\title{
Comparison of HCMV IE and EF-1 $\alpha$ Promoters for the Stable Expression of $\beta$-Subunit of Hexosaminidase in CHO Cell Lines
}

\author{
Incilay Sinici, ${ }^{1,3}$ Maryam Zarghooni, ${ }^{2}$ Michael B. Tropak, ${ }^{2}$ \\ Don J. Mahuran, ${ }^{2}$ and H. Asuman Özkara ${ }^{1}$
}

Received 11 May 2005-Final 20 October 2005

Published online: 28 April 2006

\section{INTRODUCTION}

The ability to use in vitro mutagenesis and transfection techniques to generate stable cell lines that highly express a mutant protein associated with a metabolic disease has been an important technique in linking genotype to clinical phenotype (Özkara and Sandhoff, 2003). One group of metabolic diseases is GM2 gangliosidosis (AB-variant, Tay-Sachs and Sandhoff diseases). These diseases are characterized by the accumulation of GM2 ganglioside, primarily in the lysosome of neuronal tissue, leading to progressive neurodegeneration. Tay-Sachs and Sandhoff diseases are caused by defects in the HEXA gene, which encodes the $\alpha$-subunit, and the HEXB gene, which encodes the $\beta$-subunit of heterodimeric $\mathrm{N}$-acetyl- $\beta$-hexosaminidase (Hex) (EC 3.2.1.52), respectively (Gravel et al., 2001; Mahuran, 1999). The AB-variant is associated with defects in the GM2 activator protein, a substrate-specific cofactor for Hex A. The second major Hex isozyme in normal human tissue is Hex $\mathrm{B}$, a homodimer of $\beta$-subunits.

In this study, we introduced a cDNA encoding the $\beta$-subunit of human Hex $(\beta$ cDNA) into three different eukaryotic expression vectors containing two different promoters, in order to establish which produces the highest expression levels of Hex B activity and $\beta$-protein in permanently transfected $\mathrm{CHO}$ cells. Our results indicate that the type of promoter employed can be critical in the establishment of highly expressing cell lines.

\footnotetext{
${ }^{1}$ Department of Biochemistry, Faculty of Medicine, Hacettepe University, 06100 Ankara, Turkey.

${ }^{2}$ Research Institute, Hospital for Sick Children, Toronto, Canada.

${ }^{3}$ To whom correspondence should be addressed; e-mail: isinici@hacettepe.edu.tr.
} 


\section{MATERIALS AND METHODS}

\section{Construction of Plasmids}

Three different mammalian expression vectors were used; pIRES2-EGFP and pcDNA3.1D/V5-His-TOPO contained the HCMV IE promoter, and pEFneo carried the $\mathrm{EF}-1 \alpha$ promoter.

$\beta$ cDNA (1747 bp) was generated by PCR using primer-a $\left(5^{\prime}\right.$ CACCATGGGCTGTGCGGGCGGGGCTGC-3'), which encodes the Kozak sequence with a start codon, and primer-b (5'-CCTCCA'ITTT TTACATGTTCTCATG-3'), which contains the $3^{\prime}$ stop codon. To lower the PCR mutation frequency, KOD High Fidelity (HiFi) polymerase (Novagen, Japan), which has a $3^{\prime} \rightarrow 5^{\prime}$ exonuclease activity, was used. PCR was performed in a total volume of $50 \mu \mathrm{L}$ as previously described (Sinici et al., 2004). PCR products were separated on a $1 \%$ agarose gel and purified by QIAquick gel extraction kit (Qiagen, Canada).

The generated $\beta \mathrm{cDNA}$ was first cloned into pCR2.1-TOPO vector to facilitate cloning into pIRES2-EGFP vector (Clontech, Canada), according to instructions of the TOPO TA Cloning Kit (Invitrogen, USA) protocol. The $\beta$ cDNA was excised from pCR2.1-TOPO by digestion with XhoI-BamHI. The $\beta$ cDNA was then ligated into the XhoI-BamHI digested pIRES2-EGFP vector. This vector coexpresses enhanced green fluorescent protein (EGFP) and allows monitoring of the transfection and selection efficiencies of cells by using fluorescence microscopy. It places the $\beta$ cDNA under direct transcriptional control of the HCMV IE promoter.

The $\beta$ cDNA was next cloned into the pcDNA3.1D/V5-His-TOPO (Invitrogen) vector based on the instructions of the Directional TOPO Expression Kit manual (Invitrogen). The $\beta$ cDNA in pcDNA3.1D/V5-His-TOPO vector is also under the control of HCMV IE promoter. The pcDNA3.1D/V5-His-TOPO/lacZ vector, encoding $\beta$-galactosidase, was cotransfected as a control.

The third construct was prepared using pEFneo vector (made by Dr. Anson) (Anson et al., 1992). Once the $\beta$ cDNA was inserted, by the digestions of BamHINotI site derived from pcDNA3.1D/V5-His-TOPO vector carrying the $\beta \mathrm{cDNA}$, its expression was driven by the EF- $1 \alpha$ promoter, i.e., the human protein elongation factor promoter.

The three different constructs were transformed into competent $E$. coli cells by One Shot TOP10 Chemical Transformation Kit (Invitrogen). To check the transformation efficiency, we transformed one vial of One Shot Top10 cells with $10 \mathrm{pg}$ of pUC19 using the manufacturer's protocol. Clones were isolated by QIAprep Kit (Qiagen).

All constructs were confirmed by restriction digestions and sequencing analyses (ACGT Corp., Toronto). 


\section{Cell Culture and Transfection}

Chinese hamster ovary (CHO) cells were grown in $\alpha$-Minimal Essential Media (MEM) with $10 \%$ fetal calf serum (FCS), penicillin, and streptomycin at $37^{\circ} \mathrm{C}$ in $5 \% \mathrm{CO}_{2}$. Ten micrograms of each vector was transfected to the $\mathrm{CHO}$ cells $(40 \%$ confluent) using Superfect Transfection Reagent (Qiagen). After $48 \mathrm{~h}$, the cells were trypsinized and diluted ten-fold in $\alpha$-MEM plus FCS containing $600 \mu \mathrm{g} / \mu \mathrm{L}$ neomycin. Following 2 weeks growth in drug-containing media, stably transfected neomycin-resistant cells were harvested, and their Hex activity and $\beta$-proteinlevels determined. Stably transfected (pIRES2-EGFP), living CHO cells were also monitored for their expression of the EGFP reporter gene using a Leica DMIRB fluorescence microscope (Canada).

\section{Hex Activity Assay}

Transfected CHO cells were lysed in $10 \mathrm{mM}$ sodium phosphate buffer, $\mathrm{pH} 6.0$, and $5 \%$ glycerol containing a protease inhibitor cocktail (Calbiochem, Canada), by six freeze-thaw cycles. Another set of transfected $\mathrm{CHO}$ cells was treated in the same manner, with the additional use of proteasome inhibitors lactacystin and kifunensine (Sigma, USA). Protein from cell lysates was quantitated (Bio-Rad, USA) (Bradford, 1976) and the Hex and $\beta$-glucuronidase activity levels were determined using the fluorogenic substrates 4-methylumbelliferyl-2-acetamido-2-deoxy- $\beta$-Dglucopyranoside (MUG) and 4-methylumbelliferyl beta-glucuronide, respectively (Brown and Mahuran, 1991). For CHO cells transfected with pcDNA3.1D/V5His-TOPO, $\beta$-galactosidase activity in lysates was assayed as a transfection efficiency control (Zhang et al., 2000). All experiments were repeated three times in duplicate.

\section{Western Blot Analysis}

Samples of transfected and nontransfected $\mathrm{CHO}$ cell lysates, carrying equal $\beta$ glucuronidase activities, were analyzed by polyacrylamide gel electrophoresis in sodium dodecylsulfate by Laemmli gel system (12.5\% gel) using the BioRad mini-gel system (Laemmli, 1970). Western Blot analyses were performed as previously described (Sinici et al., 2004) and protein bands visualized according to the protocol recommended for the Amersham ECL system (Amersham).

\section{RESULTS}

Prior to transfections, the nucleotide sequences and orientation of the $\beta \mathrm{cDNA}$ insert were confirmed in all three constructs (data not shown). After 2 weeks of neomycin selection, nontransfected control cells died, and antibiotic-resistant 

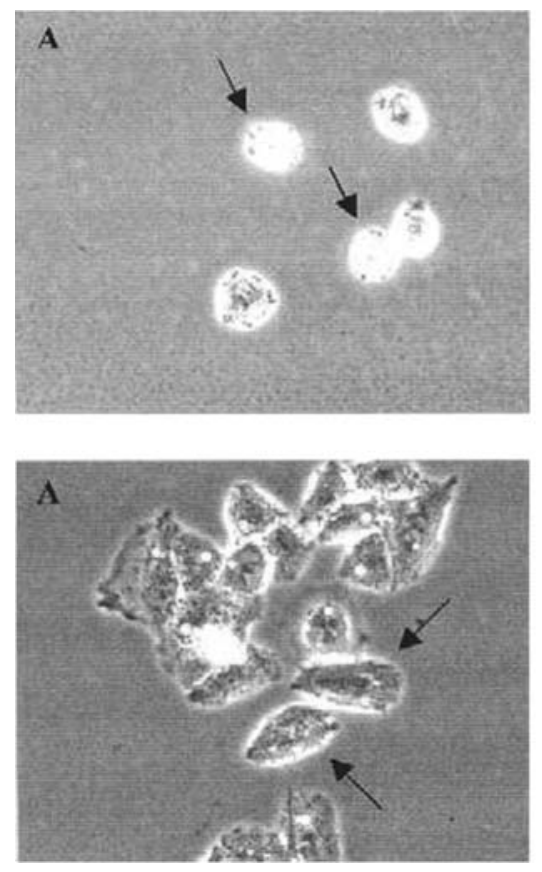

Fig. 1. A: CHO cells were visualized under light microscope. B: EGFP expressions were visualized using fluorescence microscope. Arrows indicate the cells having EGFP expressions. Upper figures show mock, lower figures show $\beta$ cDNA-inserted pIRES2-EGFP transfected CHO cells.

colonies, transfected with the pIRES2-EGFP vector, expressed the EGFP reporter gene (Fig. 1). In other cells transfected with the pcDNA3.1D/V5-His-TOPO/lacZ construct, significant neutral $\beta$-galactosidase activity was observed (Table I). Hex activity levels were then determined (Table I). The resultant specific activities of Hex from the cells transfected with the $\beta$ cDNA, inserted into either the pIRES2EGFP or pcDNA3.1D/V5-His-TOPO vectors, were very low, nearly identical to the endogenous levels in mock transfected cell lysates. The inclusion of proteasome inhibitors had no effect on these low levels of Hex activity (data not shown). When the pEFneo construct was used, the human Hex activity was found to be $\sim 1.8$ times higher in $\beta$ cDNA inserted pEFneo transfected cells than in mock transfected cells (Table I).

Extracts from mock and $\beta$ cDNA-transfected $\mathrm{CHO}$ cells were analyzed by Western Blotting using rabbit anti-human Hex B that does not cross-react with endogenous $\mathrm{CHO}$ cell Hex (Fig. 2). Although EGFP expression was observed in CHO cells transfected with pIRES2-EGFP, $\beta$-subunit proteins (pro $\beta$ and mature $\beta$ forms) could not be detected by Western blot analysis. The same lack of detectable $\beta$-protein was seen in the pcDNA3.1D/V5-His-TOPO transfected cells. However, 


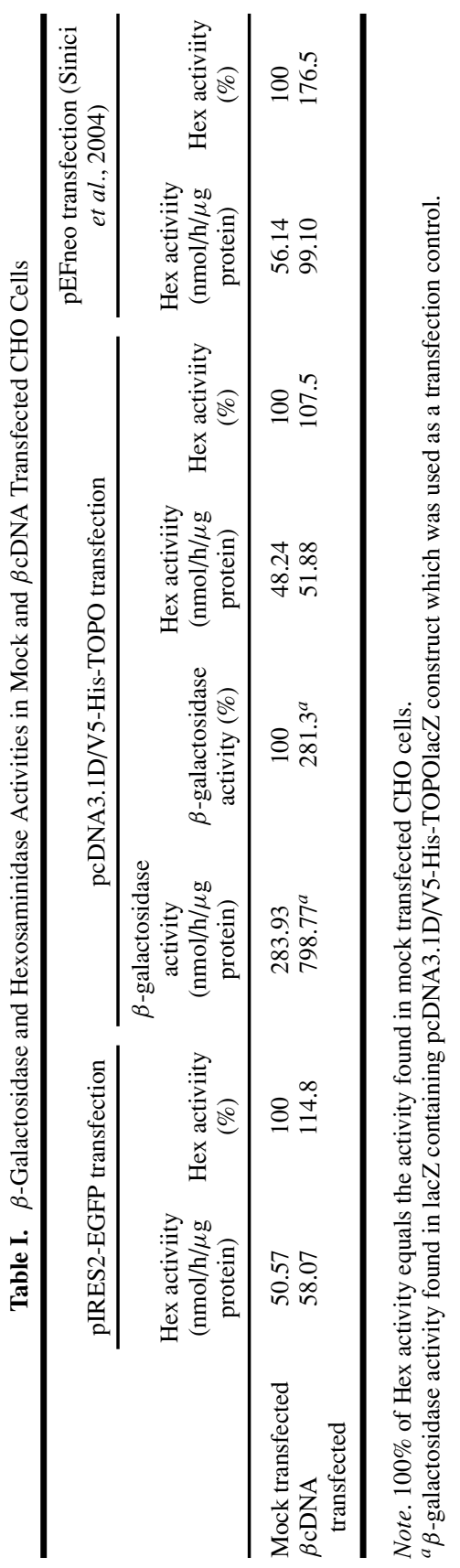




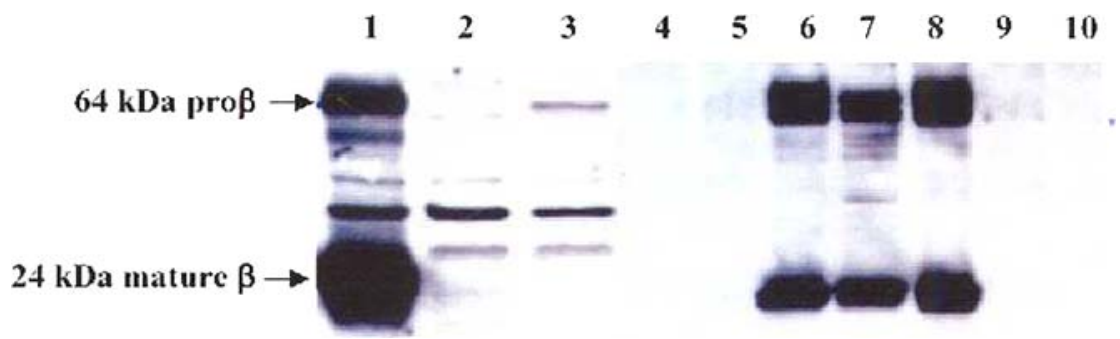

Fig. 2. Western Blot analysis of pEFneo, pIRES2-EGFP, and pcDNA3.1D/V5-His-TOPO transfected $\mathrm{CHO}$ cell lysates. The positions of the pro $\beta$ and mature $\beta$ protein bands are indicated by arrows. Lane 1: $(+)$ control; CHO cell lysates transfected with $\beta$ cDNA inserted pEFneo. Lane 2: $(-)$ control; mock-transfected $\mathrm{CHO}$ cell lysates. Lane 3: CHO cell lysates, transfected with $\beta$ cDNA inserted pIRES2-EGFP. Lane 4: ( - ) control; mock-transfected CHO cell lysates that were treated with lactacystin. Lane 5: ( - ) control; mock-transfected $\mathrm{CHO}$ cell lysates that were treated with kifunensine. Lanes 6 and 8: $(+)$ control; $\mathrm{CHO}$ cell lysates transfected with $\beta \mathrm{cDNA}$ inserted pEFneo that were treated with lactacystin. Lane 7: $(+)$ control; $\mathrm{CHO}$ cell lysates transfected with $\beta$ cDNA inserted pEFneo that were treated with kifunensine. Lane 9: CHO cell lysates transfected with $\beta \mathrm{cDNA}$ inserted pcDNA3.1D/V5-His-TOPO that were treated with lactacystin. Lane 10: CHO cell lysates transfected with $\beta$ cDNA inserted pcDNA3.1D/V5-His-TOPO that were treated with kifunensine.

extracts from pEFneo-transfected cells contained both a strong pro $\beta$-chain band and the processed mature $\beta$-chain band, consistent with a high level of $\beta$ cDNA expression in these cells (Sinici et al., 2004).

\section{DISCUSSION}

This study examines the relative effectiveness of the two common promoters in expressing the $\beta$-subunit of Hex. In an attempt to get a high level of expression of $\beta$-subunits in $\mathrm{CHO}$ cells, we inserted the $\beta \mathrm{cDNA}$ into two new generation vectors pIRES2-EGFP and pcDNA3.1D/V5-His-TOPO, each carrying the HCMV IE promoter, and the older $\mathrm{pEFneo}$ vector, carrying EF-1 $\alpha$ promoter. Interestingly, in neomycin-resistant cells transfected with vectors containing the HCMV IE promoter, no significant $\beta$-subunit protein or increase in Hex $\mathrm{B}$ activity was detected. Expression of the $\beta$-subunit (activity and protein) was observed only when the $\beta$ cDNA was under the control of the EF- $1 \alpha$ promoter.

Others have reported differences in expression of heterogeneous genes under EF-1 $\alpha$ and HCMV promoters in certain cell types (Gopalkrishnan et al., 1999; Kim et al., 2002; Teschendorf et al., 2002; Tokushige et al., 1997). Chung et al. (2002) found that the EF promoter was 200-300 times stronger than the HCMV promoter in driving the expression of the humanized renilla green fluorescent protein (hrGFP) gene. In another report, the EF-1 $\alpha$ promoter was found to be 3-fold more active than the HCMV promoter in transfected CHO cells (Kim et al., 2002). As well, the expression of cDNAs under the EF- $1 \alpha$ promoter was reported 
to be unaffected by the passage number or time elapsed between introduction and integration of the plasmid DNA. It is possible that with time, $\mathrm{CHO}$ cells may develop methods of suppressing the HCMV IE promoter. It has been suggested that expression driven by the EF- $1 \alpha$ promoter would be relatively independent of cell type and difficult to suppress, because of its indispensable housekeeping function in all mammalian cells (Goldman et al., 1996; Kim et al., 1990).

Furth et al. (1994) reported, based on histochemical analysis, that the cDNA expression driven by the HCMV IE promoter was highly variable, even in the same cell type. Teschendorf et al. (2002) described a scattered or mottled pattern of expression of the green fluorescence protein (GFP) in stable cell lines transfected by vectors carrying HCMV promoter. On the other hand, EF- $1 \alpha$ promoter gave rise to a remarkably homogeneous cell population expressing the GFP gene. We observed a similar situation in our neomycin-resistant cells expressing the EGFP reporter gene (Fig. 1). Thus the expression levels driven by the HCMV IE promoter can vary considerably even from cell to cell within a stably transfected cell line.

We conclude that any inexplicable failure to establish cell lines stably expressing a cDNA of interest, such as the $\beta$-subunit of Hex, may be due to promoter selection. Certainly, in our case the HCMV IE promoter was not the best choice, and by switching to an expression vector utilizing the human EF- $1 \alpha$ promoter, we significantly increased the level of expression of the $\beta$-subunit of Hex in $\mathrm{CHO}$ cells.

\section{ACKNOWLEDGMENTS}

We thank Amy Leung for excellent technical assistance. This work was supported by the Scientific and Technical Research Council of Turkey (TUBITAK).

\section{REFERENCES}

Anson, D. S., Taylor, J. A., Bielicki, J., Harper, G. S., Peters, C., Gibson, G. J., and Hopwood, J. J. (1992). Correction of human mucopolysaccharidosis type-VI fibroblasts with recombinant $N$-acetylgalactosamine-4-sulphatase. Biochem. J. 284:789-794.

Bradford, M. M. (1976). A rapid and sensitive method for the quantitation of microgram quantities of protein utilizing the principle of protein-dye binding. Anal. Biochem. 7:248-254.

Brown, C. A., and Mahuran, D. J. (1991). Active arginine residues in beta-hexosaminidase. Identification through studies of the B1 variant of Tay-Sachs disease. J. Biol. Chem. 25:15855-15862.

Chung, S., Andersson, T., Sonntag, K. C., Bjorklund, L., Isacson, O., and Kim, K. S. (2002). Analysis of different promoter systems for efficient transgene expression in mouse embryonic stem cell lines. Stem Cells 20:139-145.

Furth, P. A., St Onge, L., Boger, H., Gruss, P., Gossen, M., Kistner, A., Bujard, H., and Hennighausen, L. (1994). Temporal control of gene expression in transgenic mice by a tetracycline-responsive promoter. Proc. Natl. Acad. Sci. U.S.A. 91:9302-9306.

Goldman, L. A., Cutrone, E. C., Kotenko, S. V., Krause, C. D., and Langer, J. A. (1996). Modifications of vectors pEF-BOS, pcDNA1, and pcDNA3 result in improved convenience and expression. Biotechniques 21:1013-1015. 
Gopalkrishnan, R. V., Christiansen, K. A., Goldstein, N. I., DePinho, R. A., and Fisher, P. B. (1999). Use of the human EF-1alpha promoter for expression can significantly increase success in establishing stable cell lines with consistent expression: A study using the tetracycline-inducible system in human cancer cells. Nucleic Acids Res. 27:4775-4782.

Gravel, R., Kaback, M. M., Proia, R. L., Sandhoff, K., Suzuki, K., and Suzuki, K. (2001). The GM2 gangliosidoses. In Scriver, C. R., Beaudet, A. L., Sly, W. S., Valle, D. (eds.), The Metabolic and Molecular Bases of Inherited Disease, 8th edn., McGraw-Hill, New York, pp. 3827-3876.

Kim, D. W., Uetsuki, T., Kaziro, Y., Yamaguchi, N., and Sugano, S. (1990). Use of the human elongation factor 1 alpha promoter as a versatile and efficient expression system. Gene 91:217-223.

Kim, S. Y., Lee, J. H., Shin, H. S., Kang, H. J., and Kim, Y. S. (2002). The human elongation factor 1 alpha (EF-1 alpha) first intron highly enhances expression of foreign genes from the murine cytomegalovirus promoter. J. Biotechnol. 93:183-187.

Laemmli, U. K. (1970). Cleavage of structural proteins during the assembly of the head of bacteriophage T4. Nature 15:680-685.

Mahuran, D. J. (1999). Biochemical consequences of mutations causing the GM2 gangliosidosis. Biochem. Biophys. Acta 1455:105-138.

Özkara, H. A., and Sandhoff, K. (2003). Characterization of two Turkish beta-hexosaminidase mutations causing Tay-Sachs disease. Brain Dev. 25(3):191-194.

Sinici, I., Tropak, M. B., Mahuran, D. J., and Özkara, H. A. (2004). Assessing the severity of the small inframe deletion mutation in the $\alpha$-subunit of $\beta$-hexosaminidase A found in the Turkish population by reproducing it in the more stable $\beta$-subunit. J. Inherit. Metab. Dis. 27:747-756.

Teschendorf, C., Warrington, K. H. Jr., Siemann, D. W., and Muzyczka, N. (2002). Comparison of the EF-1 alpha and the CMV promoter for engineering stable tumor cell lines using recombinant adeno-associated virus. Anticancer Res. 22:3325-3330.

Tokushige, K., Moradpour, D., Wakita, T., Geissler, M., Hayashi, N., and Wands, J. R. (1997). Comparison between cytomegalovirus promoter and elongation factor-1 alpha promoter-driven constructs in the establishment of cell lines expressing hepatitis C virus core protein. J. Virol. Methods 64:73-80.

Zhang, S., Bagshaw, R., Hilson, W., Oho, Y., Hinek, A., Clarke, J. T., and Callahan, J. W. (2000). Characterization of beta-galactosidase mutations Asp332 $\rightarrow$ Asn and Arg148 $\rightarrow$ Ser, and a polymorphism, Ser532 $\rightarrow$ Gly, in a case of GMI gangliosidosis. Biochem. J. 348:621-632. 